\title{
The Structures of Perception: An Ecological Perspective
}

\section{Michael James Braund}

\author{
The seemingly paradoxical assertion will be made that \\ perception is not based on sensation. That is, it is not \\ based on having sensations... but it is surely based on \\ detecting information - James J. Gibson. ${ }^{1}$
}

$\int$ ames J. Gibson is one of the best known and perhaps most controversial visual theorists of the twentieth century. Writing in the vein of the American functionalists, and immersed in their profound sense of pragmatism, Gibson sought to establish a more rigorous foundation for the study of vision by reworking its most fundamental concepts. Over the five decades of his distinguished career, Gibson brought new clarity to the old problems of the tradition. He offered an alternative theory of perception one that could accommodate the experimental insights of contemporary research programs. He characterized this new theory as a version of direct perception in order to distinguish it from the traditional indirect approach of Rene Descartes. On Descartes' account, our perceptual awareness of reality comes through the representations we have formed of it within ourselves. In contrast, Gibson's theory of direct perception states that the environment contains all of the information needed to specify its properties. Hence, perceiving these properties is a matter of detecting the information available in the environment. This theory avoids the difficulty of explaining how the mind organizes holistic perceptions from atomic sensations. In what follows, I will attempt to make good these claims by situating the concept of "structure" at the heart of the ecological program. I will argue that this concept is significant precisely because it allows Gibson to locate the moving, perceiving body at the heart of meaningful perceptual experience; an experience which emerges in the dynamical structures that cross the body and the world.

In section one, I will distinguish Gibson's concept of the environment from the Cartesian notion of the material world. This step is necessary in any account of visual perception. The environment must first be described, since what there is to be perceived must be stipulated before one can talk about perceiving it. According to Gibson, the concept of the world can be analyzed at different levels. Gibson does not wish to give an analysis of the world at the level of Cartesian physics, which reduces the world to extended matter and

${ }^{1}$ James J. Gibson, The Senses Considered as Perceptual Systems (Boston: Houghton Mifflin Company, 1966), 1. 


\section{THE STRUCTURES OF PERCEPTION}

perpetual movement (guaranteed by the circular motions of vortices). Gibson is concerned with the world at the level of ecology, in which animal and environment form an integrated system of mutual constraint. To draw this distinction, Gibson offers (what I call) a structural analysis of the environment. This amounts to an explanatory scheme based on principles of selforganization. This analysis leads Gibson to formulate the concepts of the meaningful environment and the perceiver-environment coupling he calls reciprocity. In contrast, Descartes's mechanistic analysis "does not lead naturally to the perceiver-environment concept." 2 This is because mechanistic analyses explain things in terms of general laws established in advance of the particular things being studied. In this sense, Descartes's mechanistic analysis is consonant with his scientific ideal of objectivity. This ideal leads Descartes to conceive things in terms of a fully constituted, objective world governed by fixed laws that transcend it. In opposition, the ecological conception of structure is dynamic through and through. The structure of the environment does not consist of solid parts and a ready-made skeleton of laws that control parts (from the outside). Rather, structure emerges within a web of movement that spins between the perceiver and the environment. This multiplicity of movements forms a moving structure that is the ongoing result of the very movements that are so structured. I will argue that Gibson's ecological approach avoids the pitfalls of Cartesian dualism, by rooting perceptual theory in the concept of perceiver-environment reciprocity.

In section two, I provide an in-depth analysis of the structures of the information that is perceived in the environment. For visual perception, the information is light. However, the term "light" is ambiguous. "Light" means different things in different sciences, and the textbooks are not at all clear about the distinctions. Thus, I will examine what I take to be the central thesis of ecological optics, namely, that light in an environment is structured and (therefore) carries information for perception. The claim is highly controversial. When Descartes published the Dioptrics in 1637, he laid the foundations for the (now) orthodox view that light as such is internally devoid of perceptual information. Therefore, in order to facilitate a discussion on the claim that light carries information for perception, I will utilize Descartes's theory of light. In this section, I will delve deeper into Gibson's concepts and prepare the conceptual ground upon which a theory of direct perception can stand.

In section three, I provide an analysis and interpretation of Gibson's theory of affordances (the core of the Ecological Approach). Based on the arguments presented in previous sections, I will argue that the theory of affordances shows that perception is more than a means of passively representing the intrinsic physical organization of objects. Perception is inherently active and exploratory. It is seeks out alterations in the vast flow of information enveloping it. These alterations are detected when the perceiver moves through the environment and probes it with a pair of glancing eyes.

2 James J. Gibson, The Ecological Approach to Visual Perception (New Jersey: Lawrence Erlbaum Associates, Inc., 1979), 8. 
Locomotion opens up new possibilities for the pick-up of information specific to the perceiver's environment. This information can then be used to guide subsequent movements, as in a perception-action loop (the two being inseparable). As David Morris puts it, "we do not ... perceive naked properties of the environment, rather we perceive what the environment affords to our bodies, what we can do with, or in the environment." 3 The theory of affordances demonstrates that the structures of information are intrinsically meaningful for perceptually-guided action.

In section three, I will also argue that there is information in the environment to specify affordances, thereby linking the theory of affordances with the structural analysis of direct perception offered in the first two sections. On my interpretation, the two are inseparable. Affordances are not subjective valuations superimposed on sensations (as theories of perception typically presuppose). Rather, the affordances of the environment are directly perceived as structural information in the environment.

\section{Section 1: The Structure of the Environment}

Ever since Descartes, psychology has been held back by the doctrine that what we have to perceive is the "physical" world that is described by physics. I am suggesting that what we have to perceive and cope with is the world considered as the "environment."

As this quote indicates, Gibson is primarily concerned with a description of the world at the level of ecology, that is, a description of the environment. The Gibsonian concept of the environment is a radical departure from the traditional Cartesian theory of the material world. Since the time of Descartes, the view that the world is composed of extended, uniform matter in mechanical interaction has been an unchallenged axiom of western science. On this view, material objects are located in a container of space. Their locations are specifiable with reference to the three Cartesian coordinates, and also along an abstract dimension of time. At this mechanistic level of analysis, the various properties of the world are described in terms of their intrinsic physical organizations (i.e., texture, size, shape, mass, reflectance, chemical composition, etc.). In turn, these properties are conveyed to the perceiver via mechanical interactions among bits of matter. ${ }^{5}$ The visually perceivable

${ }^{3}$ David Morris, The Sense of Space (New York: State University of New York Press, 2004), 13.

${ }^{4}$ James J. Gibson, quoted in Edward Reed, James J. Gibson and the Psychology of Perception (New Haven: Yale University Press, 1988), 279).

5 On the Cartesian account, the perceiver's body (including the senses) is regarded as an extremely complex object of the material world. Gibson writes, "The animal is thought of as a highly organized part of the [material] world but still a part and still an object." The Ecological Approach to Visual Perception, 8. Gibson rejects the Cartesian notion of the body as one material object among others. According to Gibson, this way of thinking neglects the importance of the "perceiver-environment reciprocity" (Ibid.) concept discussed in this paper. 


\section{THE STRUCTURES OF PERCEPTION}

properties of objects are conveyed by light energy, which gives rise to visual experience. Tactile properties, such as solidity and texture, and sounds emitted from objects are conveyed mechanically and then represented as tactual and auditory experiences.

In this respect, Descartes's mechanical description of the material world is also the basis of his theory of perception. For Descartes, perception is the causal outcome of a linear chain of events beginning in the material world and (paradoxically) ending in the mind. The first event in the chain of occurrences is a physical stimulus making contact with the photoreceptive retina(s) in the material eye(s). This contact initiates a succession of nervous impulses in the sensory channels, followed by a cascade of impulses along receptor tracts to the brain. For Descartes, the resulting pattern of sensoryneural activity gives rise to meaningful perception indirectly, that is, through the performance of mental inferences. On this account, perceptual meaning is an inferred property. It is imposed on the world by the perceiver's mental constructions, rather than being a quality discovered in the world. Just as there is no place for color or taste qualities in the material world, there is also no place for the quality of meaning. Cartesian matter is literally meaningless. Given these assumptions, Descartes theorizes that perceptual meaning is part and parcel of inner representations (which are distinct from the domain of the material world). This causal account of how meaningful perceptions are constructed reflects Descartes's more general application of mechanical models to explain all perceptual processes, bodily functions and natural occurrences.

Expressing himself in this dualistic way leads Descartes into a thicket of problems. The main problem presented by his approach is the problem of mind-world interaction. If world and mind are different in substance, then the interaction between them would be of an "imposed nature." 6 This is because experience belongs to an unextended mind, but refers to extended matter. Thus, in perceptual experience, the mind's inferences cross a gap between ideas and things, which are two very different sorts of substances. To account for this phenomenon, Descartes must explain how the physical properties of things in the world are disclosed to the psychical entities (i.e., ideas) in the mind. Descartes offers a mechanical theory of indirect perception, in which "independent physical objects mechanically impart effects from one to another" in a linear fashion. This mechanical model is sufficient to explain how physical stimuli impart motion to the eye and brain of the perceiver, since stimuli, eyes, nerves and brains are embedded in a common material substance (the spatiality of which conforms to the axioms of Cartesian geometry). However, explaining how "influences are passed between qualitatively different entities, such as a physical energy causing an experiential quality [like] a sensation" in the purely psychical mental substance of a mind, is impossible on the basis of Descartes's standard causal account. The question still remains

${ }^{6}$ Harry Heft, Ecological Psychology in Context (New Jersey: Lawrence Erlbaum Associates Inc., 2001), 126.

${ }^{7}$ Ibid., 126.

${ }^{8}$ Ibid. 
as to how properties of the world are experienced by the perceiver. Thus, Descartes is faced with the problem of interaction, which he addresses in the Meditations.

This is the conceptual morass that Gibson's ecological program was designed to circumnavigate. To overcome the difficulties inherent in Cartesian dualism, Gibson's analysis of the environment is concerned with structures rather than mechanisms. Roughly, a structural analysis of the environment centers on the individual's continuous transactions with meaningful features of the environment. At this level of analysis, individuals engage the environment in order to learn more about its properties and, in many cases, to contribute to the environment's changing structural character. This analytical stance emphasizes the reciprocity of the environment and the perceiver. On this view, the environment (to be perceived) does not reside in the representations of an individual's mind. If it did, then an unbridgeable gap would exist between the "real" environment and its cognitive representation in the mind.

In visual theory, the term structure was first introduced by Gestalt psychologists. For the Gestalts, a structure is a form or standard that fits changing content to an already specified framework. For instance, Koffka is primarily concerned with how we see an object in relation to its framework, that is, as a figure on a background. He argues that the figure-background framework constitutes the "intrinsic structure" of the visual field. In other words, the figure-background configuration is a template that bundles changing content into pre-specified forms or gestalts. As David Morris points out, "it fits the contingent to the necessary, the fluid to the stable, or the a posteriori to the a priori." The Gestalt conception of the figure-background configuration can accurately be described as an a priori structure of perception that organizes the fluctuating, a posteriori visual contents by fitting them to pre-specified forms.

From the ecological perspective, this notion of structure is problematic. Difficulties spring up when we ask how changing content calls a new structure into play, since this would require a structure for applying structure. This is problematic because it results in an infinite regress on the task that a structure is meant to achieve. What is required, then, is a concept of structure that is not detached from what it structures. Hence, this concept is neither a priori nor a posteriori. It cannot be interpreted within this conceptual division, which overlooks the crucial role played by the reciprocal movements of perceivers and the environment in structural analysis.

The concept of structure is difficult to articulate because our daily engagement with the environment gears us to put solid things before moving processes, and (consequently) mechanisms before structures. We are, as Henri Bergson points out, inclined to the logic of solids. ${ }^{10}$ For this reason, theories typically decompose structure into solid parts in an a priori framework of mechanical laws that control parts. This is the basic thrust of Descartes's

\footnotetext{
${ }^{9}$ Morris, The Sense of Space, 34.

${ }^{10}$ Ibid., 80.
} 


\section{THE STRUCTURES OF PERCEPTION}

mechanistic analysis of the material world. In contradistinction, Gibson resists the temptation of reducing structure to mechanism by conceiving structure as a process or movement that manifests its own self-organizing pattern within a perceiver-environment system. If we had to say what the moving structure is made of, or what it is the organization of, we would have to say that it is the self-organization of movement that is animated through perceiver-environment reciprocity. I will return to this point shortly.

To further elucidate the distinction between structural and mechanistic analyses, consider the example of a traffic jam. ${ }^{11}$ A mechanistic analysis would reduce a traffic jam into independent, vehicular units governed by universal laws of traffic. For Gibson, the mechanistic analysis overlooks the fact that traffic jams would not occur if people drove in a law-like manner at all times. Traffic movement does not follow from mechanistic laws. Commenting on this example, David Morris writes, "Different rules of driving emerge in moving contexts that drivers collectively create." 12 He continues, "Experientially, this is quite palpable. There is a different way to drive in smooth highway traffic versus a highway traffic jam. There are subtle differences between traffic jams, which do not result from a priori laws, but from interaction in a given web of moving traffic."13 Thus, whereas a mechanistic analysis aims at explaining things in terms of general laws (i.e., laws established in advance of the particular things being studied), structural analysis studies the perceiver-environment system, which actively organizes itself through the reciprocal movements within this system.

Therefore, unlike mechanistic analyses, the ecological approach does not seek universal laws that cover all possibilities of mechanical interaction. Qua ecological, this approach concerns the manner in which the structure of the perceiver-environment system, through the dynamic interrelations of its parts, constrains possibilities. ${ }^{14}$ This is the goal of dynamic systems theory, a recent development in ecological optics, which regards moving structures as rooted in constraint formations. To return to Morris's example, cars never behave as independent units. In a highway-traffic system, like the TransCanada, automobile movement is inherently limited by the road and by the moving interrelation of cars, buses and trucks. Not all movements are possible. As Turvey and Carello point out, this amounts to a collapse of the degrees of freedom of a system. ${ }^{15}$ In this respect, highway-traffic systems are not causal outcomes of all-encompassing mechanical laws. They are structured by constraints that are immanent within the actual movement of the highwaytraffic system.

\footnotetext{
${ }^{11}$ Ibid., 56.

12 Ibid.

${ }^{13}$ Ibid.

${ }^{14}$ The concept of "constraints" was introduced into visual theory by dynamic systems theorists. In my paper, it serves as a conceptual apparatus for facilitating an understanding of the dynamics of perceiver- environment systems.

15 M.T. Turvey and C. Carello, "Dynamic Touch," in Perception of Space and Motion
} (California: Academic Press, 1995). 
These "immanent constraints"16 are due, not merely to the arrangement of inert bits of matter in mechanical interaction, but to the organization of movement animated within the perceiver-environment system. For Gibson, constraints arise in moving structures that open the perceiver to the environment and the environment to the perceiver reciprocally. The collapse of the degrees of freedom of a perceiver-environment system is itself brought about through the limitations intrinsic to reciprocal movements within this system. This is why dynamic systems theorists, following Gibson, tell us that moving structures can be understood in terms of constraint formations. The dynamic organization of this system is generated by the reciprocal interrelations of its constituents (i.e., perceivers and environments) which, in turn, place constraints on the range of the functioning of these constituents. In other words, the degrees of freedom of the constituents of the perceiverenvironment system are constrained by the dynamic organization of the system as a whole. To illustrate this point, consider Carello and Turvey's example of wielding a hand held object, like a baseball bat. ${ }^{17}$ Carello and Turvey argue that one's feeling for the length of a wielded object, such as a bat, has to do with one's possibilities for movement, or more specifically, with the way those possibilities are constrained by the joint activity of the body and the bat. Felt length does not refer to a geometrical length reconstructed by an inferential process in the mind (a la Descartes); it refers to something directly within the moving interaction of the perceiver and the environment. In the language of dynamic systems theory, this something is called the "eigenvalue of the inertia tensor of the object."18 The eigenvalue of the inertia tensor is a set of values that specifies the difficulty in moving something about, i.e., its wieldiness. ${ }^{19}$ On this account, the felt length of the bat corresponds to its wieldiness. Importantly, wieldiness can only be detected when the bat is taken in hand and moved around in a specific way. The point is that, what we are perceiving when we perceive felt length or wieldiness is a set of constraints or limitations on movement that organize themselves within the perceiver-environment system. The joint activity of the body and the bat constrains or limits the degrees of freedom of its wielder. In line with Gibson, Carello and Turvey conclude that all individual actions are constrained and hence organized within this system as a whole.

Gibson's structural analysis leads him to formulate the concept of the environment, not as a container of entities, but as dynamic structures that manifest in perceiver-environment reciprocity. A possible objection to Gibson is that this definition fails to answer the following question, namely, "What is the environment, this concept that supposedly overcomes the dualism of Descartes's mechanistic analysis?" If Gibson cannot respond, it seems he is pulling a rabbit out of a hat.

\footnotetext{
${ }^{16}$ Morris, The Sense of Space, 69.

${ }^{17}$ C. Carello, and M.T. Turvey, "Rotational Invariants and Dynamic Touch," in Touch, Representation and Blindness (Oxford: Oxford University Press, 2000).

18 Turvey, and Carello, "Dynamic Touch."

${ }^{19}$ Morris, The Sense of Space, 22.
} 


\section{THE STRUCTURES OF PERCEPTION}

I am suggesting this objection is poorly put. It assumes that Gibson is seeking a fixed entity called "the environment." But such a definition would conflate structure and mechanism and thus return us to Descartes's problems. Against this tendency, Gibson argues that the environment is an open system in which dynamic, self-organizing structures manifest in a moving circuit across purposive actions and shared behavioral settings. This is a subtle, but vast shift in perspective. Whereas Descartes conceives the material world as a totality of physical entities that (somehow) interact with psychical entities via preestablished laws, Gibson abandons the interactionist approach and its assumptions. For this reason, Gibson does not pursue an independent conception of the environment isolated from the embodied perceiver. $\mathrm{He}$ writes, "The terms animal and environment make an inseparable pair. Each term implies the other. No animal could exist without an environment surrounding it. Equally, although not so obvious, an environment implies an animal ... to be surrounded." 20 Thus, the structures of the environment do not transcend the perceiver-environment system (as mechanical laws transcend the material world they govern). Rather, structures emerge from within the very systems they constrain.

The ecological approach discussed in this paper has several advantages over the mechanistic approach and the representational theory of perception it engenders. First of all, the concept of perceiver-environment reciprocity avoids the dilemma of (private) mind-(public) world interaction by conceiving perceivers and environments as forming a mutually supportive system. Moreover, the ecological approach establishes this conception without reducing the problem to physical-neurobiological terms, as do contemporary mechanistic approaches. In truth, this reductive turn simply dresses the old Cartesian problem in a modern garb. This is the problem of how properties in the material world are actually experienced by perceivers. For this reason, theories that reduce perceptual experience to neurobiological processes do not avoid the problem of interaction. In contrast, Gibson's ecological program is designed to overcome these difficulties by rooting the perceiver and the environment in a fully integrated system of mutual constraint, instead of treating them as two distinct domains of existence in (inexplicable) mechanical interaction.

These introductory remarks have enormous import for ecological theories of perception (discussed in the proceeding sections). Behind these remarks is a sustained attack on Descartes's mechanical theory of perception and the perceived world. To recapitulate, this theory states that physical stimuli initiate patterns of excitation in the sensory receptors on the retina, yielding collections of "immediately" experienced sensations in the material body. On this account, perception is the "mediate" procedure of constructing representations on the basis of these elementary, atomic sensations. In opposition to this view, Gibson argues that perception is wrongly conceived as a private event in the perceiver's mind. Representational theories of perception

${ }^{20}$ Gibson, The Ecological Approach to Visual Perception, 8. 
appeal to frameworks fixed in advance of the perceiver's moving, ecological relation to the environment. Models that transcend the perceiver-environment system are both problematic and unnecessary. The structures generated in perceiver-environment systems serve as "stimulus information" 21 for the direct perception of the environment. In Gibson's terminology, perception is the detection of invariant structures in the flow of stimulus information, which is sparked when the active perceiver moves through the environment. This is the topic of the next section. In discussing the structures of the information for perception, I will draw on many of the insights in this section.

\section{Section 2: The Structure(s) of Information in the Environment}

As I understand it, ecological optics analyzes the information for vision. This analysis consists of two components. The first is a structural analysis of the environment to be perceived and the information available in the environment. The second component is a similar analysis of how perceivers detect and use the information available to them. As I have suggested, these two components are complementary. Environmental information is itself a product of the perceiver's bodily involvement with the environment. This interaction generates a "flow" of information, which underlies and guides all perceptual activity. Hence, the dynamic encounter of the moving perceiver and the environment is the basis of the theory of direct perception.

The viability of a theory of direct perception depends on demonstrating that there is information in the environment that is capable of specifying its source. Hence, to claim that environmental information does not need to be reconstructed or embellished by inferential processes is to claim that stimulation is uniquely and invariably tied to its source. Like all branches of optics, ecological optics claims that light is the stimulus for vision. Only light can meaningfully stimulate the eye of the observer. However, the claim that light in the environment is structured and that these structures serve as information about the environment is controversial. The reason for this, Gibson explains, is that "It has been asserted with some plausibility both that light carries information about the world and that it does not." 22 This debate is as old as the history of visual theory itself. On the one hand, theorists as historically remote as Ptolemy and Kepler have argued that (1) objects constantly send off little images of themselves in all directions and that these images serve as the active information for perception. This was the dominant view until Descartes produced his radical critique of the doctrine of intentional species in The World and the Dioptrics. 23 Thereafter, it became a foregone

\footnotetext{
${ }^{21}$ Ibid., 52.

22 Gibson, The Senses Considered as Perceptual Systems, 186.

${ }^{23}$ The doctrine of intentional species holds that the lens of the eye acts as a selector of visual information and transforms the physical stimuli of light and color into visual impressions. These, in turn, give rise to perceptual images that are passed back along the stream of visual spirits
} 


\section{THE STRUCTURES OF PERCEPTION}

conclusion that (2) nothing gets into the eye but the rays of light, propagated from "points" in geometrical space. Cartesian light rays are perfectly homogenous "pencils" of moving particles (and therefore lack any internal structure needed to carry semblances of visible qualities of the object to the eye of the perceiver). Upon entering the eye, the light rays converge to a series of points on the retina. Each point in the stimulated region mechanically produces a sensation from which the mind infers determinate properties of the external world. This is the basis of Descartes's theory of indirect perception.

In rejecting (1) are we forced to accept (2)? That is, if we deny that light carries information for perception insofar as it transmits an exact replica of the object to the eye, must we assume that light is internally devoid of information? If so, then we must also be prepared to accept the claim that the mind builds up a representation of the world from the momentary sensations caused by the stimulation of points on the retina. According to Gibson, this theory is needlessly complex and attributes too much responsibility to the inferential status of the intellect. ${ }^{24} \mathrm{I}$ am suggesting that the difficulties inherent in this view can be overcome by Gibson's doctrine of ecological specificity.

In this section, I will put forward a version of Gibson's theory as an alternative to representationalism by contrasting the Cartesian and Gibsonian models of light and stimulation. This contrast is a conceptual tool for highlighting the innovations of the ecological approach. For example, whereas Descartes's theory of vision regards the perceiver as a monocular being, with one immobilized eye fixed passively toward impoverished optical displays, Gibson argues that ordinary perception (which seldom occurs under conditions that mimic the controlled experimental settings of laboratory psychophysics) is nothing like this. Human perceivers are mobile beings, who actively engage the world and explore it with a pair of moving eyes. In accounting for the role played by activity in perception, Gibson's theory of "ambient light" emphasizes the structures of information generated at a moving point of view. These structures serve to constrain and guide all perceptual explorations. Since "the essence of ecological optics is the demonstration that there is information in ambient light" 25 for active perceivers, I will begin with the question, "What is ambient light?" My answer will consist of a general description of ambient

(traveling mediators) to local brain centers. These images serve as representations of their generating objects, enabling both perception and cognition. For Descartes, however, there is no internal structure in light itself that carries semblances of visible qualities of the object to the eye of the perceiver. Since signs need not "resemble the things they signify," as is shown by the case of letters, words, and sentences, such an internal structure in light is not necessary. Images and sensations are not "out there" in the world, flittering about and being transmitted externally. In Descartes's philosophy, there are no ideas outside of the mind. Thus, by conceiving light as motion Descartes eliminates the Scholastic's intentional species "flittering through the air" from thing to mind and any other theory that claims the visible is a phenomenon that constitutes itself outside of us.

${ }^{24}$ Here Gibson is employing the law of parsimony (also known as Occam's Razor). The law states that, all else being equal, one should prefer simple theories or explanations over complex ones.

25 James J. Gibson, "Ecological Optics," in Reasons for Realism: The Selected Essays of James J. Gibson (New Jersey: Lawrence Erlbaum Associates, 1982), 73. 
light and an analysis of the structures of the information it contains for an active perceiver.

In The Ecological Approach, Gibson describes ambient light as "light reflected by the environment as a source." 26 The layout of the environment is primarily composed of a transparent medium and a nested set of textured surfaces. The medium transmits light, whereas the surfaces reflect it diffusely. ${ }^{27}$ These surfaces reflect in multiple fashions, from one surface to another, and to yet another. The outcome of diffuse, multiple reflections is an omnidirectional flux of light, which fills the transmitting medium and envelopes the perceiver. If this light has different intensities in different directions, instead of the same intensity in all directions, Gibson proposes to call the flux of light an ambient optic array. It is an array because the variation of intensities produces a differential arrangement within the total field of structured light. It is an optic array because the differential arrangement, constituted by the variations in intensity, provides an active, mobile perceiver with information about the environment. Visual perception is a matter of detecting this information within the ambient optic array. On this account, perceptual information is intrinsic to the environment, instead of being intrinsic to sensations in the perceiver's mind. Consequently, the appeal to inferences is unnecessary. This is because perception is not a matter of inferentially reconstructing or representing the world on the basis of sensory intermediaries (as Descartes assumes). No mediation is required. Rather, perception is a form of direct contact with the ambient optic array and the information contained therein.

As I mentioned, the issue of whether light contains a perceptible structure signifies a major difference between the Cartesian and Gibsonian approaches to vision. Whereas Descartes's conception of light (as a stimulus) consists of individual rays propagated from "points" in geometrical space to corresponding points on the retinal plane, Gibson's ambient optic array does not consist of individual rays. According to Gibson, a ray is a geometrical fiction. While "such a fiction may be useful for geometrical optics, and convenient for the tracing of rays through refracting media," 28 it cannot provide the visual system with information about the environment. A ray of light is unstructured; it is perfectly dense and vanishingly thin. However, if light is to carry information about the environment it must be structured, otherwise nothing can be made visible on the basis of light. ${ }^{29}$ This claim holds in general. Any space filled with unstructured light is as devoid of information as a fog-filled medium. Gibson writes, "[In a fog-filled medium] the air would be translucent but not transparent . . Multiple reflections would occur only between closely packed microsurfaces, yielding a sort of microillumination of

${ }^{26}$ Gibson, The Senses Considered as Perceptual Systems, 186.

27 In a typical environment, surfaces reflect light diffusely, not regularly, since mirrors are rare in nature.

28 Gibson, "Ecological Optics," 66.

${ }^{29}$ In this respect, a light ray vanishing to a geometrical line in Cartesian space would offer nothing to be seen. 


\section{THE STRUCTURES OF PERCEPTION}

things too small to see." 30 Light without structure is similar to absolute darkness. It would provide no information about the environment. In this respect, Gibson offers (what I have called) a structural analysis of ambient light. The structures of ambient light emerge in the dynamic interaction of the moving perceiver and the environment. These structures constitute information for an active perceiver because of her direct, environmental contact with the ambient optic array.

The structure of the ambient optic array is comprised of two complementary components. Gibson calls these the invariant and perspective structures, respectively. When the perceiver moves through the environment, the interaction of the moving body against the field of light generates a kind of flow in the ambient optic array. In ecological optics, the flowing character of the array is what makes perception possible. Gibson's central insight is that perception is the detection of invariants that emerge within the flow as the perceiver moves. Invariants refer to higher-order relations in the structure of ambient light that do not change in the context of an otherwise changing array. These invariants of the ambient optic array constitute its essential structure, that is, these are what tend to persist despite change. However, what is invariant in the array does not emerge "except within a flux of variation." Gibson continues, "The essentials become evident in the context of the changing nonessentials," 31 meaning that persistence and change are co-dependent terms. Gibson calls the latter the perspective structure of the array, which governs the "perspective transformations... in the ambient optical field." 32 It is a moving structure. The invariant structure, on the other hand, constitutes the optical pattern that persists despite the changes of perspective structure. There are many such invariants in a typical environment, the most important of which is the horizon that separates the ground from the sky. It is the invariant of invariants, so to speak, acting as the limit of all gradients of texture density in the array. The closer something appears to the horizon, the closer it is to reaching its limit of perspective minification. This is what is meant when something is described as "vanishing in the distance."

Of course, invariants of structure can be detected at every level of the persisting environment. In the Ecological Approach, Gibson considers the problem of how a "rectangular surface like a tabletop can be given to sight when presumably all that an eye can see is a large number of forms that are trapezoids." 33 This is a variation on the argument from illusion, as presented by George Berkeley. According to Gibson, the argument is a pseudo-dilemma; it is only a problem if we assume that form-perception is an intellectual synthesis of discrete sensory-retinal inputs. This is the approach taken by Descartes and Berkeley. ${ }^{34}$ However, for Gibson, the trapezoidal forms on the retina are of no

\footnotetext{
${ }^{30}$ Gibson, The Ecological Approach to Visual Perception, 52.

31 Ibid., 73.

32 Gibson, The Senses Considered as Perceptual Systems, 195.

${ }^{33}$ Gibson, The Ecological Approach to Visual Perception, 74.

${ }^{34}$ For a detailed discussion see M.J. Braund, "The Indirect Perception of Distance: Interpretive Complexities in Berkeley's Theory of Vision," in KRITIKE: An Online Journal of
} 
account, since we do not perceive a succession of retinal images. It is their transformation that counts as the perspective structure, and the invariant structure (i.e., the rectangularity of the tabletop) is revealed by these transformations. Gibson writes, "Although the changing angles and proportions of the set of trapezoidal projections are a fact, the unchanging relations among the four angles and the invariant proportions over the set are another fact, equally important, and they uniquely specify the rectangular surface." 35 Thus, the invariant structure of the array is only revealed when the observer is permitted to move and the perspective structure flows.

Crucially, the flowing transformations of the perspective structure and the underlying invariant structure are concurrent. Invariants are patterns of change, which emerge when the perceiver moves around. Consider Morris's phenomenological experiment in support of this claim. He writes, "If you immobilize yourself in a room and unfocusedly gaze at a ceiling corner, you might perceive that corner as flattening out into three lines meeting at an angle, but the moment you sweep your eyes back and forth, the angles flow through transformations" 36 (my italics). The invariant pattern of these transformations specifies the persisting features of the environment. Accordingly, Gibson characterizes invariants as patterns of movement in the ambient optic array. What is invariant is the pattern of variation. To clarify this point, Morris offers the example of a waltz. "The waltzing dancers move around in all sorts of cycles, but the pattern of these cycles (i.e., the basic one-two-three step, the way these steps go in a circle, etc.) remains invariant." 37 For Gibson, perception is the detection of invariant patterns that emerge within the reciprocal movement that plays back and forth across the perceiver and the environment. Thus, invariant information specifying the persisting features of the environment is generated through perceiver-environment interaction (as in the case of moving around an object). This is made possible, in part, by bodily locomotion.

Locomotion plays a central role in any information-based theory of perception. In this theory, perception and action are invariably coupled, for if perception is to acquire information from the structured array, the perceiver must be capable of movement. Movement generates optical flow and reveals the invariant patterns that underpin it. This information serves to guide embodied action in the environment. It informs us of those aspects of the environment that play a role in the pragmatic organization of our activities. This information can then be used to guide subsequent movements in a perception-action loop. On this view, the function of vision is not the production of mental representations, but rather, the enabling of the perceiver to function appropriately in the environment. Gibson conceives of vision as a way of acquiring information about the environment by coming into direct

Philosophy, 1:2 (December 2007), 49-64, < http://www.kritike.org/journal/issue 2/braund december2007.pdf $>$.

${ }_{35}$ Gibson, The Ecological Approach to Visual Perception, 74.

${ }^{36}$ Morris, The Sense of Space, 61.

${ }^{37}$ Ibid., 61. 


\section{THE STRUCTURES OF PERCEPTION}

contact with it via active explorations. After all, "The first responses of a man to an optic array . . . are to focus, fixate, modulate its intensity, and above all explore it." 38

The term "exploration" suggests that perception is more than a means of passively receiving light into the body. Vision is a way of looking, that is, a way of moving influenced by what we see. Gibson shows how vision depends on movement and the way that seeing depends on looking. Gibson writes,

Looking around and getting around do not fit into the standard idea of what visual perception is. But note that if an animal has eyes at all it swivels its head around and it goes from place to place. The single, frozen field of view provides only impoverished information about the world. The visual system did not evolve for this. The evidence suggests that visual awareness is panoramic and does in fact persist during long acts of locomotion. ${ }^{39}$

Elsewhere, he explains that exploration of the ambient optic array is possible because its structure constitutes "a global stimulus rather than a punctate stimulus." 40 According to Gibson, the former is information for perception, whereas the latter is not. The ambient optic array is a global stimulus because it fills the transmitting medium of the environment and surrounds the perceiver. It is extended, enduring, and cannot be given all at once. This goes along with the conviction that perception is a temporal act. It unfolds over time as the "active, exploring observer" 41 moves about her surroundings and samples the array in successive overlapping sectors. Conversely, a "punctate" stimulus is momentary, like a pinprick. It is given "all at once" and does not permit exploration..$^{42}$

On Gibson's view, Cartesian light rays are punctate stimuli that resemble the stimulus prods used in traditional, laboratory psychophysics. When applied to an eye, the individual rays touch off a pattern of "points" on the retina. Since light rays are unstructured and do not carry information about their source, Descartes theorizes that perceptual information is encoded in the array of sensations triggered by the incident rays. This gives the impression that the senses are passive channels for sensations, i.e., mechanisms for passively receiving imposed stimuli and transmitting the attendant sensations to various brain centers vis-à-vis the optic nerves. Upon reaching their cerebral

\footnotetext{
38 Gibson, "Ecological Optics," 64.

${ }^{39}$ Gibson, The Ecological Approach to Visual Perception, 2.

40 Gibson, "Ecological Optics," 64.

41 William H. Warren Jr., "Self-Motion: Visual Perception and Visual Control," in Perception of Space and Motion (California: Academic Press, 1995) 264.

${ }^{42}$ In section three I will return to this idea of exploration, which signifies the deep coupling of perception and action. On Gibson's account, "exploration" is just a synonym for the activity of utilizing perceived affordances. This activity has its informational support in the ambient optic array, which I will also discuss in section three.
} 
destinations, the sensations are decoded and become the basis of visual inferences about the world. This theory regards the "sensorium as a personal entity ... shut off from the external world but seeking information about it and perceiving the representatives ... of objects that the nerves bring to it." 43 For Descartes, it is these sensory representatives that are directly perceived, not the objects represented. Accordingly, inferential awareness becomes a necessary component of Descartes's sensation-based theory of indirect perception.

The concept of the ambient optic array is of monumental significance for Gibson because it explicitly breaks with Cartesian representationalism and the notion that vision is mediated by retinal sensations. It allows him to transcend this old way of thinking, both in his theorizing and in his experimentation. Whereas Descartes was searching for some mapping from the world to the mind (and back) in order to find out how retinal sensations represented reality, Gibson abandons this entire project and its assumptions. Such a conception of the nature of vision is pitched at the wrong level. The world may "map" onto the ambient optic array, but it need not map into the organism's mind. ${ }^{44}$ The environment (i.e., the animal's habitat) is the sole repository of information about it. Vision acquires information directly by exploring the ambient optic array with (a pair of) moving eyes. Therefore, no mediation is necessary.

In this section, I have stressed the importance of bodily motility in perception. A perceiver's use of the ambient optic array is active, not passive. Accordingly, the eye is not simply a receptacle for discrete light stimuli imposed on the retina, but rather, an organ for exploring an ambient optic array. In this respect, the visual system is responsible for obtaining stimulation over time, not merely for receiving it in momentary prods. Vision can perform this function by paying attention to whatever is invariant in the structured array. Of course, invariants are only revealed when the perceiver is permitted to move and the optical display flows through transformations. For Gibson, the transformations in the ambient optic array provide the information upon which vision is founded. On this account, perception is not based on having sensations (as traditional accounts suppose). Sensations are momentary and discrete, corresponding to nothing in actual experience. In order to account for the unity of perceptual experiences, sensation-based theories have to synthesize the momentariness of sensation with conceptual processes. These processes (i.e. inference, associative memory, visual memory traces, imagination, etc.) would then integrate all the fleeting sensations into

${ }^{43}$ E.G. Boring, Sensation and Perception in the History of Experimental Psychology (New York: Appleton Century Crofts Inc., 1942), 224.

44 To this effect, Gibson writes, "The structure of the environment at all levels of linear size is mapped into the structure of the array at all levels of angular size." Gibson, The Senses Considered as Perceptual Systems, 192. These perspective projections in the ambient optic array include information about the borders, edges, contours, margins and transitions between things in the environment. They are features of the structured array and constitute differences within that structure. This is necessarily the case, because no two adjacent surfaces will project the same intensity of light, when they are at different angles of inclination to the source from which it radiates. 


\section{THE STRUCTURES OF PERCEPTION}

representations. ${ }^{45}$ However, Gibson's experimental work on the concept of the ambient optic array shows that the basic elements of visual perception are not punctate sensations aggregating into mental representations, or even a field with figure and ground organization, as in the Gestalt theory of Koffka, Kohler and Wertheimer. Rather, ecological information is directly perceived and constitutes the basis of visual perception. Such is the conceptual framework of Gibson's anti-sensationalist, information-based theory of direct perception under the new doctrine of ecological specificity.

\section{Section 3: Direct Perception and the Structure of Affordances}

As discussed in sections one and two, what sets Gibson's theory apart from theories of indirect perception is his rejection of representationalism. Traditionally, representationalism is an "understanding of the place of mind in a world such that our only knowledge of reality comes through the representations we have formed of it within ourselves" 46 (my italics). This gives the impression that perception, like knowledge, is a subjective interpretation of the world outside of thought. Consequently, representational theories analyze perception as though it took place wholly inside the perceiver, in the private inferences of the perceivers mind. In contrast, Gibson's model shows how the psychological domain can be extended to properties of the environment, rendering its features publicly accessible and distributed across perceiver-environment systems. For exactly this reason, Gibson is committed to the idea that perception gives us a veridical encounter with a real environment. Crucially, we do not grasp the properties of the environment through representations (a la Descartes). Our perceptual grasp is direct and therefore unmediated by conceptual schemes.

In isolation, this claim may be inadequate because it overlooks what is most distinctive about the ecological approach. Gibson contends that while perceivers entertain a direct apprehension of the physical layout of surfaces, they do not perceive naked properties of the material world, stripped of all significance. Rather, we perceive what the environment affords to our bodies, that is, it shows what we can do with and in the environment. What we can do depends on us. Gibson calls these perceived features of the environment, which are there for our acting bodies, affordances. In this final section, I offer a

45 In the Critique of Pure Reason, Kant offers a similar (albeit transcendental) theory of the unity of empirical representations. According to Kant, this unification is the result of (1) the production of sensations (i.e., the matter of sensible intuitions) and (2) the application of the categories in experience (i.e., the conceptual form of the understanding, in which all sensations are necessarily cast). Without the latter there would be no unified representations, that is, no synthetic unity of the manifold of intuition, and without the former there would be nothing to be unified in representations. As Kant puts it, "Thoughts without content are empty, intuitions without concepts are blind." The Critique of Pure Reason, trans. by Paul Guyer and Allen Woods (USA: Cambridge University Press, 1997), A51/B76.

${ }^{46}$ Charles Taylor, "Merleau-Ponty and the Epistemological Picture," in The Cambridge Companion to Merleau-Ponty (USA: Cambridge University Press), 26. 
treatment of Gibson's controversial theory of affordances. Thereafter, I will contrast Gibsonian affordances with Cartesian inferences, in order to arrive at several conclusions about the nature of perception in general.

Simply put, affordances are the perceived functional properties of objects, places and events in relation to an individual perceiver. By functional properties, Gibson means that affordances specify the acts or behaviors permitted by these objects, places and events in the environment. He writes, "The affordances of the environment are what it offers ... [perceivers] . . what it provides or furnishes, either for good or ill." 47 Thus, functional properties set up the possibilities for action in the environment. A surface in the environment may be perceived as "sit-on-able" for an individual, if it meets certain criteria dictated by the specificities of this individual's body. For example, the surface must appear supportive of the individual's weight and be positioned approximately knee-high. The more a surface deviates from these criteria, the less it will be perceived as offering the relevant functional property, the affordance of sitting.

In this context, the claim that affordances are environmental properties that vary in relation to the individual perceiver is made clearer. A feature of the environment may present certain affordance possibilities for one individual, but not for another, owing to a mismatch in the functional properties of the individual and the environment. For example, a surface affords locomotion for an individual if and only if the functional properties of this individual are compatible with the functional properties of this sector of the environment. ${ }^{48}$ Carello and Michaels comment, while a wall provides the affordance of being walk-on-able for a fly, whose "feet are sufficient to cause an adhesive force to balance the downward force created by the effects of gravity on the animal's mass," 49 the ecological relation between a human and the (same) wall does not afford such a balance. Thus, a wall provides the functional property of being walk-on-able for some species but not others. In this regard, Gibson stresses the relational quality of affordances. The ecological relations between the perceiver and the environment constitute the various properties that are inherently significant to an individual perceiver in that context. Simply put, an affordance is the perceptual/behavioral meaning of these ecological relations (for a perceiver). To claim that "a chair affords sitting" or that it is "stand-on-able" says that when an individual perceives a chair, she is directly aware that chairs can be used for the performance of these (and other) actions. Therefore, the theory of affordances expresses the tight coupling of perceiving and acting for embodied perceivers.

${ }^{47}$ Gibson, The Ecological Approach to Visual Perception, 129.

48 Carello and Michaels schematize Gibson's notion of affordance as follows: "A situation or event $\mathrm{X}$ affords action $\mathrm{Y}$ for a perceiver $\mathrm{Z}$ on occasion $\mathrm{O}$ if certain relevant compatibilities between X and Z obtain." Presumably, these "relevant compatibilities" are the functional properties of environments and individual perceivers. See C. Carello and C. Michaels, Direct Perception (New Jersey: Prentice-Hall INC., 1981), 43.

${ }^{49}$ Ibid. 


\section{THE STRUCTURES OF PERCEPTION}

The Ecological Approach offers a conjoint treatment of perceiving and acting. This treatment is necessitated by the idea that perceptions and actions are always performed with reference to the same environment. If perceptions are to be useful they must be executed in the performance of effective actions on the environment. If actions are to be effective they must be constrained by direct perception. The information that specifies the environment must provide a basis for activity, and activity must provide a means for the performance of direct perception. Hence, the reciprocity of perceiving and acting is the key to understanding the relation between the theory of affordances and the theory of direct perception. To elaborate, I will consider an argument in support of this assertion made by Carello and Michaels. Thereafter, I will describe how the theory of affordances undercuts inferential models of indirect perception.

For Gibson, the primary use of perception is to provide a veridical encounter with a real environment, and to inform perceivers of those aspects of the environment that play a role in the organization of their activities. From the ecological standpoint, "The successful control of activity requires the availability of certain kinds of stimulus information" 50 in the environment. Thus, in order to cope with the environment in ways that bring about the realization of goals and pursuits, perceivers detect properties in the ambient optic array that permit the relevant behaviors. For instance, consider the relationship between the act of approaching a particular object (i.e., a tree) and its informational support in the ambient optic array. In order to approach the tree, one must act in such a way as to keep that object at the center of the expanding projection in the ambient optic array. The expanding projection "specifies approach, while the rate of expansion specifies the imminence of arrival." 51 To avoid collision with the tree the actor will have to slow down and eventually stop her gait when the tree is projected to a one-hundred and eighty degree visual angle in the ambient optic array.

This example shows the reciprocity of perception and action, and reinforces the claim that activities require an informational support wherein the ambient optic array permits the guidance of activity. As the perceiver moves, her actions open up new possibilities for the detection of information. This information can then be used to guide subsequent movements as in a perception-action loop. On this account, the perceptual control of action is not a one-way street from individual sensations to motor responses. 52 Perception directs actions in the environment. These, in turn, generate a flow of perspective transformations and enable the detection of invariant structures in ecological information. Affordances are those invariant properties in the ambient optic array that specify the meaningful dimensions of interaction the perceiver has with the environment. ${ }^{53}$ Hence, what individuals directly

\footnotetext{
${ }^{50}$ Ibid., 48.

${ }^{51} \mathrm{Ibid}$.

52 As it is in Descartes's theory, which states that the senses are mechanisms for passively receiving external stimuli which actuate reflex-based motor responses in the body.

${ }^{53}$ Gibson, The Ecological Approach to Visual Perception, 129.
} 
perceive are the affordances of events (i.e., the possibilities for meaningful action), rather than their intrinsic physical compositions.

As Carello and Michaels point out, "This is the innovation of affordances. That chairs afford sitting and cliffs afford avoiding is news to no one; but for Gibson, it is the affordance that is perceived." 54 We directly perceive what actions can be entered into with respect to the environment vis-à-vis affordances. Hence, affordances are items of our immediate visual experiences. This is a particularly striking aspect of Gibson's theory. On traditional accounts, meaning is considered to be a function of abstract thought, and therefore, as fundamentally distinct from the information perceived. The concept of the affordance is different, insofar as it suggests that meaning is a distinctive feature of (direct) perceptual experience and the environment. But meaning is a vague term. It is crucial to distinguish between the perceptual meaning of affordances and the conceptual meaning associated with philosophical and scientific concepts. ${ }^{55}$ This distinction is introduced by Heft in an attempt to clarify Gibson's position. Affordances are a part of the flow of perceptual experience, and are specified by ecological information generated by an active perceiver. By contrast, concepts are abstractions from the flow of perceptual experience. They are the result of second-order, cognitive processes. This is another way of saying that "affordances are directly perceived," whereas "concepts are derived and categorical." 56 Categorization is not a necessary precondition for the direct perception of affordances. Categorical thinking is abstracted from direct perceptual experience; it is a way of conceptualizing it and creating concepts that are ultimately derived from it. As Gibson puts it, "You do not have to classify and label things in order to perceive what they afford." 57

However, inferential accounts make exactly this kind of mistake. They confuse perceptual with conceptual meaning by making the former a species of the latter. This is a classic example of the experience error. In this instance, the experience error is the fallacy of transforming the information for perception (the perceptual meaning which refers to the environment as it figures for an individual perceiver that interacts with it) into ideational contents organized through a conceptual framework in the mind. Theories of indirect perception presuppose the existence of these cognitive processes, which supposedly have the function of structuring meaningful, perceptual experience from meaningless sensations via concepts. Importantly, the concepts are fixed in advance of the perceptual experiences they give rise to. As a result, the perceptual meanings of these experiences always refer back to the concepts qua organizational.

To put it differently, theories of indirect perception assume that "bits of sensation are interchangeable, determinate independent of their context,

\footnotetext{
${ }^{54}$ Carello and Michaels, Direct Perception, 42.

${ }_{55}$ Heft, Ecological Psychology in Context, 128.

${ }^{56}$ Ibid.

${ }^{57}$ Gibson, The Ecological Approach to Visual Perception, 134.
} 


\section{THE STRUCTURES OF PERCEPTION}

atomic." 58 Only perceptions can be called meaningful. However, because they also assume that perceptions are derived from sensations, just as conclusions are derived from premises, theories of indirect perception wrongly suppose that the constitution of perceptual meaning is a two-stage process of (1) acquiring meaning-neutral sensations and (2) accruing perceptual meanings to sensations vis-à-vis concepts. In previous sections, I have characterized these conceptual models as inferential accounts, which contend that the constitution of perceptual meaning is an event that occurs wholly inside the perceiver's mind.

The theory of affordances criticizes these representational theories of perceptual meaning, which appeal to conceptual frameworks fixed in advance of the perceiver's moving, ecological relation to the environment. In Gibson's estimation, theories that root perception in the a priori or the a posteriori, in deductive or inductive systems established in advance of acts of perception, are pitched at the wrong level. Perceptual meaning does not transcend the environment. Rather, perception is the detection of invariants in the flow of stimulus information generated when the perceiver moves. Such invariants specify reciprocal information about the perceiver and the environment, without any necessary appeal to organizational concepts. In this respect, the information for perceiving affordances is already present in the visual stimuli; their possibilities are ordered into the structure of the stimulus array as such. Hence, to perceive an aperture in a surface is to perceive the (possible) functional opportunity of passing through it. To perceive that a surface is level and solid is to perceive that it is "walk-on-able"59 and so forth.

The same logic applies to all higher order properties of the environment (including spatial properties). The ecological relation between the perceiver and the environment constitutes the environment as having spatial dimensions that are inherently significant. Contra Descartes, then, the organism never deals in objective measurements, which can only afford further measurements. Rather, the organism deals in "strides, striking distances and safe removes." 60 The child, frightened by a storm, does not measure the small space beneath coffee table, before taking shelter. Rather, she perceives the affordance of a safe hiding place. On the other hand, the same space does not afford hiding activities for a full grown adult (save for contortionists). Thus, the theory of affordances contains a developmental aspect, as well. Affordances are not fixed in advance of perception. Rather, they vary in relation to the individual perceiver. ${ }^{61}$

Accordingly, the available information for perceiving the separation of surfaces in depth, or surface layout, is the same information for the perception of what it affords. In the ecological approach to vision, observers do not begin

\footnotetext{
${ }^{58}$ Morris, The Sense of Space, 6.

59 James J. Gibson, "Notes on Affordances," in Reasons for Realism: The Selected Essays of James J. Gibson, 408.

${ }^{60}$ Morris, The Sense of Space, 20.

${ }^{61}$ For instance, to the toddler a rigid, vertical surface (i.e., a wall) mainly affords collision, whereas the same wall (if appropriately textured) may afford climbing for an adult.
} 
by perceiving a layout of neutral surfaces in depth. Affordances are not added to the perception of surface layout, as though they were mental contributions made by a perceiving subject. Thus, we no longer have to assume that there is (1) a sensation-based perception of a thing's shape, size, relative distance-fromhere, etc. and then (2) the accrual of meaning to the immediately perceived sensation. The information for the former is inextricable from the latter, and they are detected in exactly the same fashion. Therefore, Gibson's ecological approach surpasses the Cartesian tradition in visual theory. In the specific context of space perception, traditional accounts typically analyze depth as if it took place wholly inside the perceivers' mind. However, for Gibson, it takes place between the perceiver and the environment. Depth perception is perception of the perceiver's relation to the environment, and that relation involves life and movement. It is neither on the side of the perceiver (subjective), nor on the side of the environment (objective). It crosses between them, and is, therefore, pregnant with its own meaning. ${ }^{2}$ Gibson's theory of affordances expresses this very fact.

In conclusion, if the theory of affordances is successful, if it has explanatory power, it is because it surpasses the conceptual dialectic of traditional accounts and places perception on an entirely new footing. On Gibson's theory, visual perception is not a one-way street from retinal stimulation and the production of sensory cues to perceptual representations in the mind. This kind of model presupposes the existence of an inferential or associative process, which mediates between sensations and perceptions. I have called this indirect perception. The theory of affordances is Gibson's definitive attempt to tip the scale toward direct perception. It is supposed to prove that meaningful perception is not a subjective, mental event. Rather, meaningful perception is informed by the perceiver's bodily interaction with the structures of the environment. Affordances are higher order, relational properties of environmental information and are directly perceived on the basis of that information. As objects of direct perception, affordances emerge within and reflect perceptual activity. In this way, Gibson's theory of affordances provides both a positive account for overcoming representationalism in visual theory, and a negative account, or sustained attack, on the doctrine of atomic sensation and the use of perceptual inferences as explanatory concepts in visual theory. By making perception direct, Gibson effectively renders these concepts unnecessary.

Department of Philosophy, Brock University, Canada

\section{References}

Boring, E.G., Sensation and Perception in the History of Experimental Psychology (New York: Appleton Century Crofts Inc., 1942).

62 Maurice Merleau-Ponty, The Visible and the Invisible, trans. by Alphonso Lingis (Illinois: Northwestern University Press, 1965), 132-133. 


\section{THE STRUCTURES OF PERCEPTION}

Braund, M.J., "The Indirect Perception of Distance: Interpretive Complexities in Berkeley's Theory of Vision," in KRITIKE: An Online Journal of Philosophy, 1:2 (December 2007), 49-64, $<$ http://www.kritike.org/journal/issue 2/braund_december2007. $\mathrm{pdf}>$.

Carello, C., and Michaels, C., Direct Perception (New Jersey: Prentice-Hall INC., 1981).

Carello, C. and Turvey, M.T., "Rotational Invariants and Dynamic Touch," in Touch, Representation and Blindness (Oxford: Oxford University Press, 2000).

Descartes, Rene, Discourse on Method, Optics, Geometry and Meteorology, trans. by Paul J. Olscamp (Indianapolis: The Bobbs-Merrill Company, 1965).

Kant, Immanuel, The Critique of Pure Reason, trans. by Paul Guyer and Allen Woods (USA: Cambridge University Press, 1997).

Gibson, James J., Reasons for Realism: The Selected Essays of James J. Gibson (New Jersey: Lawrence Erlbaum Associates, 1982).

, The Ecological Approach to Visual Perception (New Jersey: Lawrence Erlbaum Associates, Inc., 1979).

The Senses Considered as Perceptual Systems (Boston: Houghton Mifflin Company, 1966).

Heft, Harry, Ecological Psychology in Context (New Jersey: Lawrence Erlbaum Associates Inc., 2001).

Koffka, H., Principles of Gestalt Psychology, trans. by Robert M. Ogden (New York: Harcourt Brace, 1935).

Kohler, W., Gestalt Psychology: An Introduction to new concepts in modern psychology, trans. by Robert M. Ogden (New York: Liveright, 1947).

Merleau-Ponty, Maurice, The Visible and the Invisible, trans. by Alphonso Lingis (Illinois: Northwestern University Press, 1965).

Morris, David, The Sense of Space (New York: State University of New York Press, 2004).

Reed, Edward, James J. Gibson and the Psychology of Perception (New Haven: Yale University Press, 1988).

Taylor, Charles, "Merleau-Ponty and the Epistemological Picture," in The Cambridge Companion to Merleau-Ponty (USA: Cambridge University Press).

Turvey, M.T., and Carello, C., "Dynamic Touch," in Perception of Space and Motion (California: Academic Press, 1995).

Warren Jr., William H., "Self-Motion: Visual Perception and Visual Control," in Perception of Space and Motion (California: Academic Press, 1995). 